\title{
Effect of Turntable Structure on Electromechanical Characteristics of Laser Tracker System
}

\author{
Erfang Cao ${ }^{1,2, a^{*}}$, Wenji She ${ }^{2, b}$, Liang Zhou ${ }^{1,2, c}$ \\ ${ }^{1}$ Xi'an Institute of Optics Precision Mechanics, Xi'an 710119, China \\ ${ }^{2}$ University of Chinese Academy of Sciences, Beijing 100049, China \\ accaoerfang@opt.cn, bshe_wj@opt.ac.cn, czhouliang@opt.ac.cn
}

Keywords: Laser Precision turntable, Electromechanical coupling system, Response speed, Cut-off frequency.

\begin{abstract}
Laser tracker is composed of a mechanical structure and a servo control system. And the characteristics of them all affect the error and stability of the laser tracker measurement system. The cut-off frequency and the response speed are important index to characterize the system performance. Among them, the cut-off frequency is the characterization of the size of the system bandwidth, and response speed is an indicator of system sensitivity. The cut-off frequency is higher, and the response speed is faster. The tracking performance of the tracker is better. Usually, when analyze the cut-off frequency of a system, various components of the control system will be the mathematical modeling, but only the mechanical structure of the inertia as a parameter into the model. It can cause errors in system analysis. In this paper, establish a mechanical and electrical model of the mechanical structure and the control structure to realize the information of the laser tracker system. Analyze the effects of rotating inertia, friction torque and structural rigidity on the characteristics of electromechanical coupling system.
\end{abstract}

\section{Introduction}

Laser tracker is a kind of precision instrument can measure the form and location tolerance and size of a large work-piece through the way of point measurement, combining of high precision laser distance measuring and angle precision measurement technology. It has high precision, fast dynamic measurement, installation, and mobile convenience and has very high application value in installation testing, large equipment in science and engineering construction, aerospace, shipbuilding, automobile, rail laying large component inspection and assembly process [1].

The tracking ability of laser tracker is determined by the frequency response bandwidth of the control system. Usually, when analyze the cut-off frequency of a system, various components of the control system will be the mathematical modeling, but only the mechanical structure of the inertia as a parameter into the model [2]. However, in practice, the friction, stiffness and moment of inertia of the mechanical structure affect the motion characteristics of the structure. A lot of ignorance of structural information will affect the accuracy of electromechanical coupling system prototype testing, but also affect the entity's test evaluation.

In this paper, establish a mechanical and electrical model of the mechanical structure and the control structure to realize the information of the laser tracker system by ADAMS and MATLAB/Simulink to realize the information of the laser tracker system. Analyze the effects of rotating inertia, friction torque and structural rigidity on the characteristics of electromechanical coupling system so as to improve the system bandwidth by changing the structure parameters.

ADAMS (Automatic Dynamic Analysis of Mechanical System) software, by the United States mechanical power company (Mechanical Dynamics Inc.), is the world's most authoritative, use the most extensive mechanical system Dynamic analysis software. ADAMS software is widely used in aerospace, automotive engineering, railway vehicles and equipment, industrial machinery, engineering machinery and other fields [3]. 
MATLAB is a matrix laboratory (Matrix Laboratory) for short. In addition to superior numerical computing capabilities, it provides professional-level symbolic computation, word processing, visual modeling and simulation, and real-time control. Simulink is an add-on component of MATLAB software and is a MATLAB software package for modeling, simulation and analysis of dynamic systems. It supports continuous, discrete and mixed linear and non-linear systems, as well as simulation systems with different sampling rates for different parts [4].

\section{Modeling}

Turntable Modeling. Laser precision tracking turntable belongs to the two-dimensional, including a horizontal shaft system, a vertical shaft system, a mounting base and an instrument bracket [5]. Establish a simplified model in order to facilitate the modeling and simulation. The damping, the housing structure the bracket and stopper mechanism are ignored. And the motor stator fixed in back-frame, encoder fixed in shaft, raster scanning head fixed in back-frame recording the rotation angle of shaft. According to the principle of movement, simplify the bearing inner ring, rotor, rotary encoders and bushings as load 1, the bearing outer ring, the reading head and stator as load 2 and the horizontal encoder and bearing as load 3. And the simplified turntable is shown in Fig.. 1.

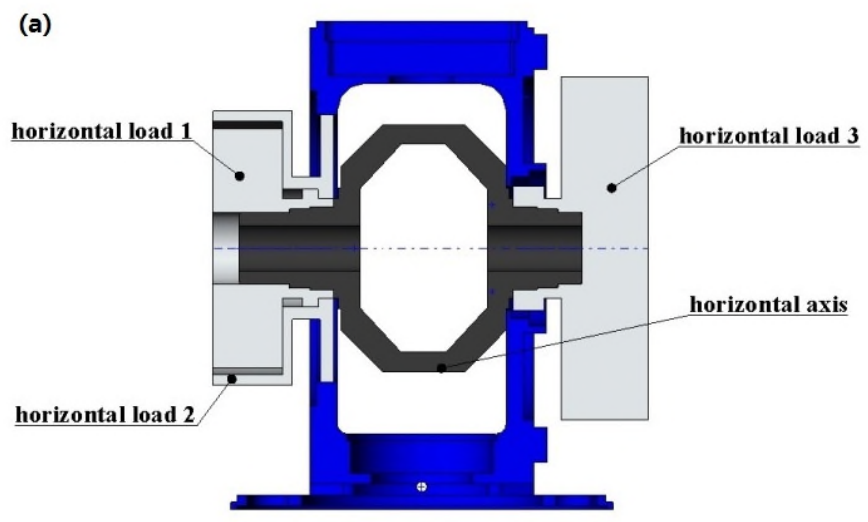

(a) simplified head

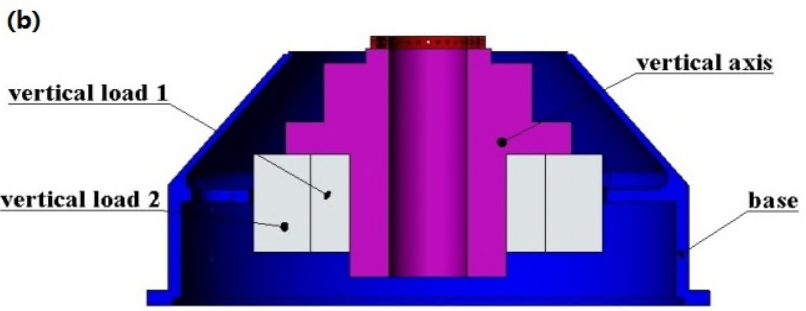

(b) simplified bottom

Fig. 1 Simplified structure.

Dynamic Modeling. Import the simplified three-dimensional model that is established in Solidworks into ADAMS, and rename each part. Define the material of each part as shown in Table 1 [6].

Table 1. Material properties of each part

\begin{tabular}{ccccc}
\hline Components & material & Density $\left(\mathrm{kg} / \mathrm{m}^{3}\right)$ & Elastic Modulus(Pa) & Poisson's ratio \\
\hline Load 1 & carbon steel & 7800 & $2.1 \times 10^{11}$ & 0.28 \\
Load 2 & titanium & 4600 & $1.1 \times 10^{11}$ & 0.3 \\
Load 3 & Stainless steel & 8000 & $1.9 \times 10^{11}$ & 0.28 \\
others & Aluminum alloy & 2700 & $6.9 \times 10^{10}$ & 0.33 \\
\hline
\end{tabular}

According to the actual movement of turntable, define the contact of horizontal load 1 and load 2, vertical load 1 and load 2 are rotating vice connection, and other parts using fixed vice connection. Table 4.2 shows the connection pairs defined by ADAMS. 
Table 2. Joints of motion between components.

\begin{tabular}{ccc}
\hline Member 1 & Member 2 & connection \\
\hline horizontal load 1 & horizontal load 2 & Rotating Vice \\
horizontal load 3 & Back box & fixed vice \\
horizontal load 1 & horizontal axis & fixed vice \\
horizontal load 2 & Back box & fixed vice \\
Unequal thickness ring & Back box & fixed vice \\
Vertical axis & Unequal thickness ring & fixed vice \\
vertical load 1 & Vertical axis & fixed vice \\
vertical load 1 & vertical load 2 & Rotating Vice \\
vertical load 2 & Base & fixed vice \\
Base & ground & fixed vice \\
\hline
\end{tabular}

According to the multi-body dynamics theory, the degree of freedom of the structural model is calculated. Each component is composed of 6 degrees of freedom. Each fixed pair constraints 6 degrees of freedom, and each rotation pair constraints 5 degrees of freedom. The degree of freedom for the assembly is [7]:

Total Freedom $=6 *$ Number of components- $6 *$ Number of fixed pair- $5^{*}$ Number of rotation pair $=6 * 10-6 * 8-5 * 5=2$

Control System Modeling. The optical tracking of the laser tracker is essentially a system of position follow-up control by detecting the offset of the laser spot on the position detector. Respectively, use three-loop feedback control structure, they are current loop, speed loop and position loop to adjust the system. The output drives the horizontal axis and the vertical axis motor, and the outgoing laser beam is always directed toward the central region of the target. The closed-loop servo control system structure is shown in Fig. 2.

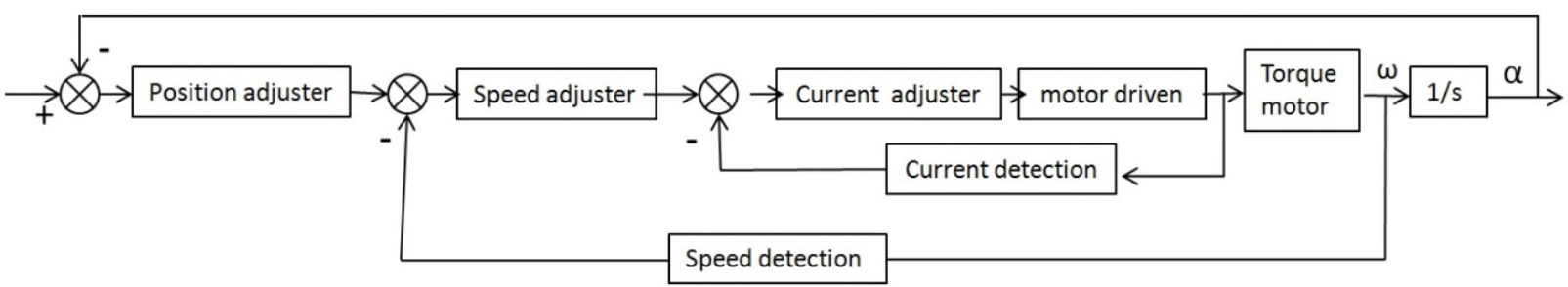

Fig. 2 The closed-loop servo control system.

Usually in the simulation of the system, the various components of the control system will be modeling, and only take the inertia of the structure into the control block diagram instead of the whole turntable. The general control block diagram of the system is shown in Fig. 3.

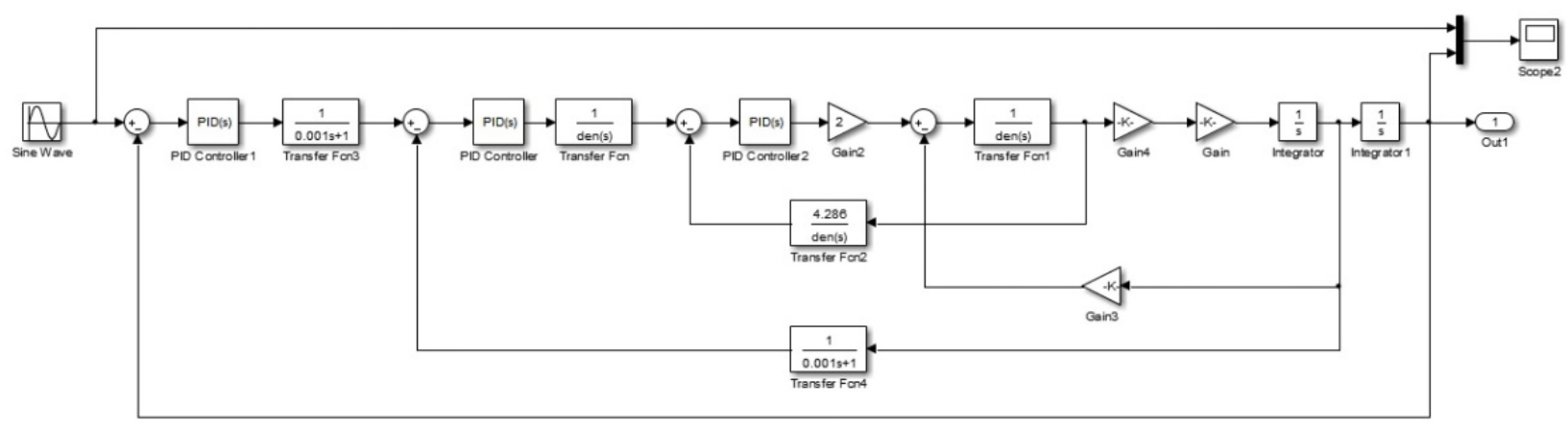

Fig. 3 The general control block diagram of the system. 
In this paper, use ADAMS software and MATLAB / Simulink software make a coupling system. ADAMS / Controls module provides the interface with MATLAB. It can achieve the dynamic link between the two platforms, mechanical and electrical integration of CNC machine tool control system simulation optimization. You need to determine the value of input and output of ADAMS through an information file or start up file in ADAMS / View or ADAMS / Solver. The output refers to the variable entering the control program, and is also the variable from the ADAMS / Controls outputs to the control program. The input refers to the variable from the control program back to ADAMS that is the output of control program [8].

\section{Electromechanical System Coupling}

In the ADAMS structure of the three-dimensional model, the following will be combined with MATLAB / Simulink control simulation [9]. Defines state variables, export the structural model and establish the electromechanical coupling model shown in Fig. 4.

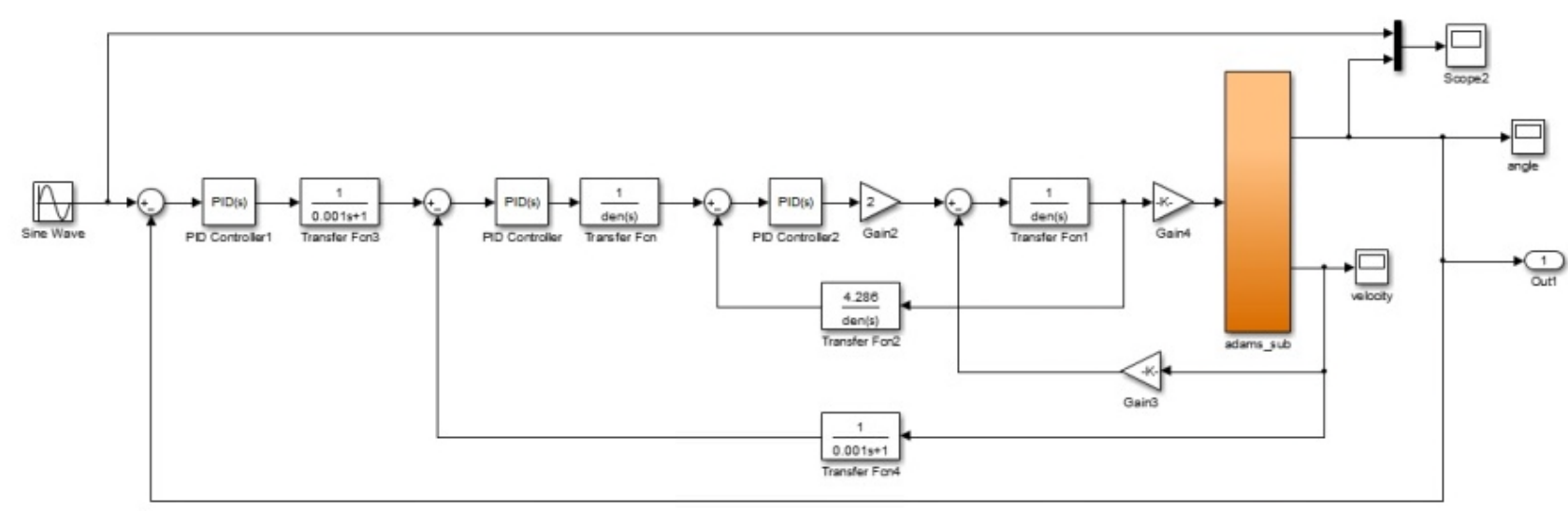

Fig. 4 Electromechanical system block diagram of turntable.

\section{Simulation and Analyze}

Influence of Turntable Structure on Coupling System. Respectively, simulate the general control system and complete the electromechanical coupling control system of the laser tracker respectively, and ensure the control parameters of the control system other remain unchanged. Enter a step signal which amplitude is 1 and a sinusoidal signal whose frequency gradually increased. The step response image and the amplitude-frequency characteristics of the two control systems are shown in Fig. 5.

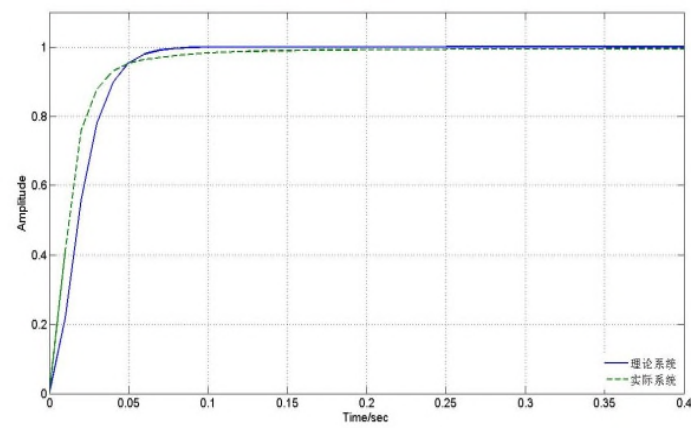

(a) Step response diagram

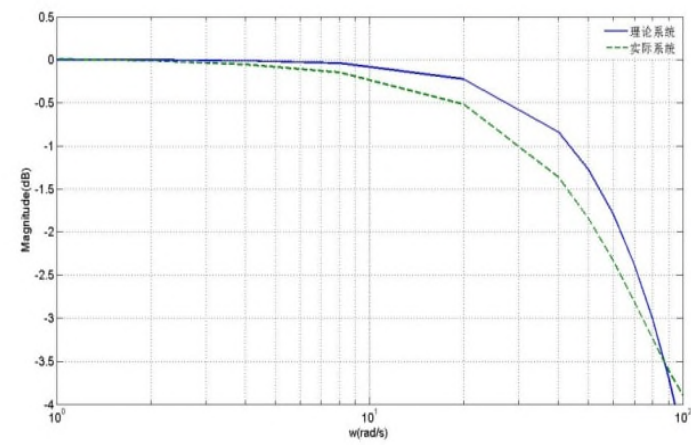

(b) Amplitude - frequency characteristic diagram

Fig. 5 Comparison of step response and amplitude - frequency characteristics of different systems

It can be seen from the figure 5 that there are significant differences between the electromechanical coupling system and only the general control system with the step-response curve and 
amplitude-frequency characteristic curve [10]. The response speed of the electromechanical coupling system to the input signal is slower than that of the ordinary system, and the cut-off frequency of the electromechanical coupling system is smaller than that of the ordinary system. Therefore, the mechanical structure of the electromechanical servo system will have an impact on the dynamic characteristics.

Influence of Moment of Inertia on Coupled System. The structure rotary inertia refers to the sum inertia of the rotating axis and the load of axis. In order to facilitate modeling and simulation, keep the same material and size of the rotation axis to rule out the influence on stiffness and friction caused by material change. Only change the size of loads to simulate the different rotary inertia. Make six different sets of moments of inertia are shown in Table 3 and the step response and system amplitude-frequency characteristics are shown in Fig. 6.

Table 3. Different models of moment of inertia.

\begin{tabular}{ccccccc}
\hline & 1 & 2 & 3 & 4 & 5 & 6 \\
\hline Moment of inertia $\left(\mathrm{kg} \cdot \mathrm{mm}^{2}\right)$ & 578.89 & 713.28 & 847.71 & 982.22 & 1116.23 & 1251.23 \\
\hline
\end{tabular}

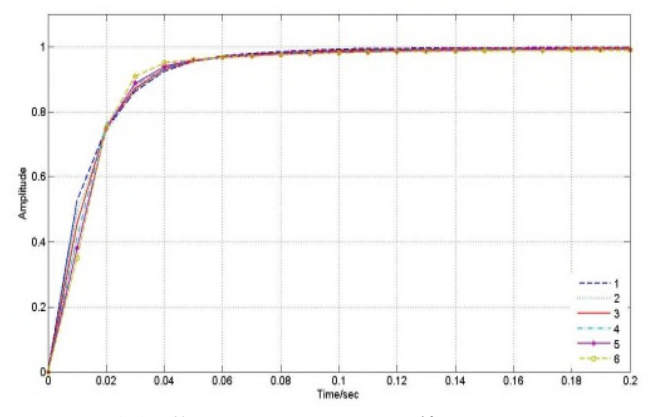

(a) Step response diagram

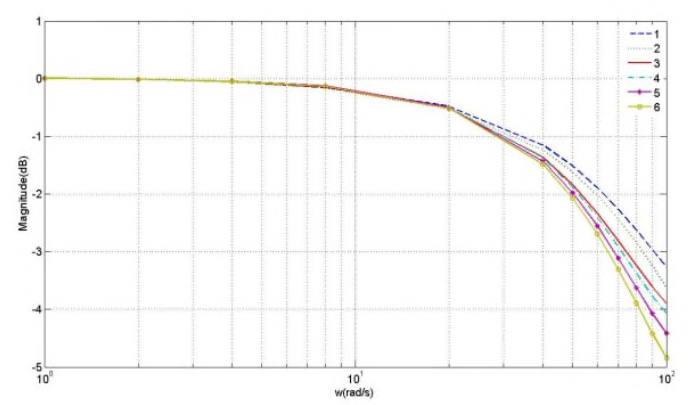

(b)Amplitude - frequency characteristic diagram

Fig. 6 The response curves of moment of inertia.

It can be seen from the figure 6 that the moment of inertia has an effect on the response speed and the cut-off frequency of the system (this can also be seen in general control system diagram). As the moment of inertia increases, the response speed and cut-off frequency of the system decrease.

Influence of Stiffness Characteristic on Coupling System. Stiffness characteristic means the ability of the material or structure to resist elastic deformation when stressed, and a characterizing of material or structure the degree of difficulty to elastic deformation. In order to facilitate modeling and simulation analysis, and to avoid the influence of the rotational inertia caused by the structural change on the natural frequency of the system, set different elastic modulus values of different materials to simulate the system of different stiffness characteristics. The elastic modulus is set to vary from $3 \times$ $10^{10} \mathrm{~Pa}$ to $1 \times 10^{11} \mathrm{~Pa}$. The analysis shows that the stiffness characteristics do not affect the dynamic characteristics of electromechanical coupling systems.

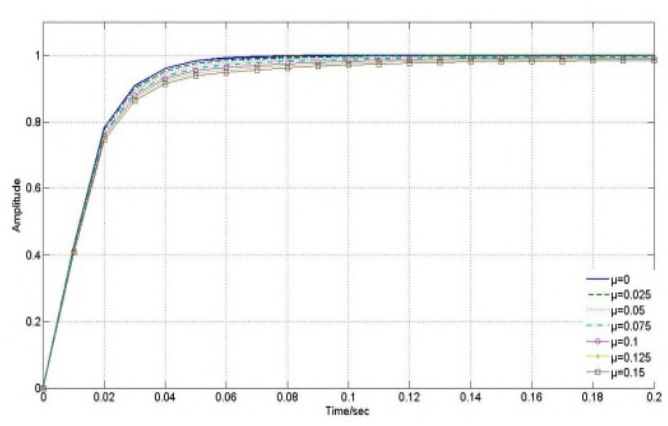

(a) Step response diagram

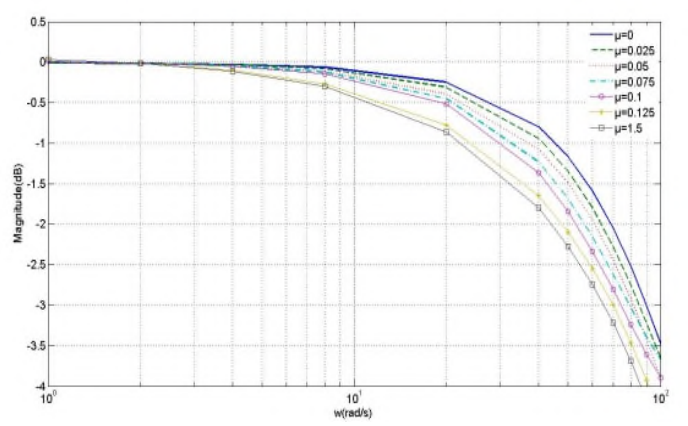

(b)Amplitude - frequency characteristic diagram

Fig. 7 The response curves for different coefficients of friction. 
Influence of Friction Moment on Coupling System [11]. Friction torque is the product of friction and friction arm. The same structure ensures the value of friction arm being constant, so simulate different friction torque by changing the friction coefficient. The friction coefficient test from 0 to 0.15 , the step size was 0.025 . The step response and amplitude-frequency characteristics of the system with different coefficients of friction are shown in Fig. 7.

It can be seen from the figure 6 that different frictional moments have different effects on response speed and cut-off frequency of the system. As the friction torque gradually increases, the response speed of the system decreases and the cut-off frequency gradually decrease.

\section{Summary}

This paper analyzes the influence of rotational inertia, stiffness and frictional moment on the characteristics of the electromechanical coupled system by simplifying and making a three-dimensional modeling of the laser precise turntable and establishing the electromechanical coupling model. The results show that the response speed and cut-off frequency of the system decrease with the moment of inertia and Friction torque increases, and has nothing to do with the material.

In order to achieve better dynamic performance of the laser tracker, in the laser precision turntable design process should be to minimize the rotation of the system part of the moment of inertia and friction torque, and also take into account the natural frequency and motor rotation characteristics and other factors.

\section{References}

[1] E. Lu, Talking about how to improve the resonance frequency of OE tracking and measuring system, Opt. Precis. Eng. 2 (1994) 47-52.

[2] H. P. Wu, X. J. Yi, K. T. Yang, Mechanism structure factors on performance of opto-electronic tracker servo system, J. Appl. Opt. 2004, 11-14.

[3] J. X. Liu, R. X. Hu, ADAMS 2012 Virtual prototype from entry to proficiency, Machinery Industry Press, 2013.

[4] G. F. Zhou, Z. X. Zhao, MATLAB/Simulink electromechanical dynamic system simulation and engineering application, Beijing University of Aeronautics and Astronautics Press, 2014.

[5] B. F. Hou, The research of 6D precision laser tracking measurement techniques, University of Chinese Academy of Sciences (Xi'an Institute of Optics and Precision Mechanics of Chinese Academy of Science), 2012.

[6] Q. S. Fan, Theoretical mechanics, Beijing: Higher education press 2014.

[7] Q. S. Fan, Mechanics of materials, Beijing: Tsinghua university press, 2014.

[8] K. Cui, Study of the dynamics coupling between the two-axis gimbal and the satellite bus (Xi' an Institute of Optics and Precision Mechanics of Chinese Academy of Science), 2013.

[9] R. R. Ma, T. Y. Wang, Dynamic coupling analysis of two-dimesional tracking turntable based on ADAMS, Journal of Changchun University of Science and Technology (Natural Science Edition), 38(1), 2015.

[10] M. D. Li, Simulation study of the electromechanical coupling facts about precise mechanical transmission in servo system (Northeastern University), 2010.

[11] Q. Ding, H. M. Zhai, The advance in researches of friction dynamics in mechanics system, Adv. Mech. 1 (2013). 